\title{
An Iterative Maximum Likelihood Synchronization Method for OFDM System
}

\author{
Chi-Min Li, Wei-Tse Sun \\ Department of Communications, Navigation and Control Engineering, National Taiwan Ocean University, Keelung, Chinese Taipei \\ Email: cmli@ntou.edu.tw
}

Received 2013

\begin{abstract}
OFDM system divides a wideband transmission bandwidth into several overlapped narrowband subcarriers to avoid the serious frequency selective fading problem. However, if Timing Offset (TO) and Carrier Frequency Offset (CFO) exist between the Transmitter (Tx) and Receiver (Rx), OFDM system will suffer the Inter-Symbol Interference (ISI) and Inter-Carrier Interference (ICI) that degrade the system performance dramatically. In this paper, we propose an iterative maximum likelihood method for synchronization. We also adopt the overlap concept to reduce the plateau problem. Simulation results show that the proposed method can predict the time delay and frequency offset correctly even under the multipath fading scenarios.
\end{abstract}

Keywords: Synchronization; Maximum Likelihood; OFDM

\section{Introduction}

Orthogonal Frequency Division Multiplexing (OFDM) has been widely adopted in the current wireless communication systems, such as IEEE $802.11 \mathrm{a} / \mathrm{g}$, and Long Term Evolution (LTE). To correctly accomplish symbol detection, an OFDM system has to establish the time and frequency synchronization [1]-[7] firstly to avoid the Bit Error Rate (BER) degradation. Poor synchronization will destroy the orthogonal property of the received signal and system will suffer the Inter-Symbol Interference (ISI) and Inter-Carrier Interference (ICI). Therefore, synchronization is a very important issue for the transmission, especially for the OFDM system.

In [3], authors proposed a Maximum Likelihood (ML) method via adopting the correlation properties of the Cyclic Prefix (CP) with the copied portion of an OFDM symbol to achieve the time and frequency synchronization. Its performance depends notably on the length of the inserted CP. The longer of the length of $\mathrm{CP}$, the better of the synchronization can be obtained. Based on this behavior, if we use a preamble with two repetitions to conduct the synchronization, the ML method should perform well. However, a serious plateau problem arises that causes the Receiver (Rx) can not predict the arrival time precisely. This problem comes from the inherent correlation properties of the preamble with two or multiple repetitions. Many methods have been proposed to reduce the plateau problem $[2,4]$.

In this paper, we propose an iterative ML synchronization method that adopts the overlap concept to reduce the plateau problem. This paper is organized as follows. In Section II, the conventional ML synchronization method and the proposed iterative method will be described in detail. Simulation results of these methods are compared in Section III. Finally, some conclusions for this paper are given in Section IV.

\section{Method Descriptions}

In OFDM system, Transmitter (Tx) can insert a CP at the Guard Interval (GI) of the OFDM signal to avoid the ISI. Assume the received signal can be expressed as

$$
r(n)=x(n-\theta) e^{j 2 \pi n \varepsilon / N}+w(n)
$$

where $\theta$ is the time delay, $\varepsilon$ is the normalized frequency offset and $w(n)$ is the Additive White Gaussian Noise (AWGN).

Consider an observation interval with $2 \mathrm{~N}+\mathrm{L}$ samples (Figure 1), the interval contains a complete OFDM symbol of $\mathrm{N}+\mathrm{L}$ samples, where $\mathrm{N}$ is the number of subcarrier and $L$ is the length of the CP.

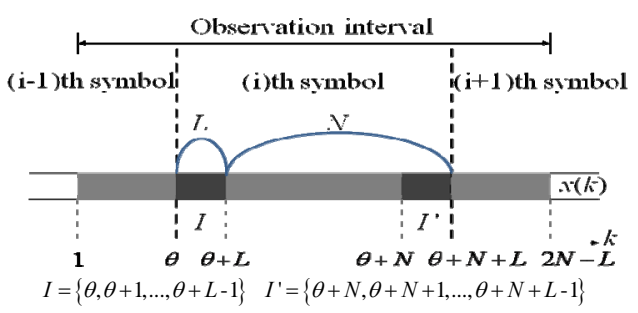

Figure 1. ML synchronization method. 
Define intervals $I$ and $I^{\prime}$ as in Figure 1, the received signal has the following correlation properties [3]:

$$
\begin{aligned}
& E\left[r(k) r^{*}(k+m)\right] \\
& =E\left[x(k-\theta) x^{*}(k+m-\theta) e^{-j 2 \pi m \varepsilon / N}\right]+E\left[x(k-\theta) e^{j 2 \pi k \varepsilon / N} w^{*}(k+m)\right] \\
& +E\left[x^{*}(k+m-\theta) e^{j 2 \pi k \varepsilon / N} u(k)\right]+E\left[u(k) w^{*}(k+m)\right] \\
& =\left\{\begin{array}{cl}
\sigma_{x}^{2}+\sigma_{w}^{2}, & m=0 \\
\sigma_{x}^{2} e^{-j 2 \pi \varepsilon}, & m=N, \forall k \in I \\
0, & \text { else }
\end{array}\right.
\end{aligned}
$$

where $\sigma_{x}^{2}=E\left[|x(k)|^{2}\right]$ is the energy of the transmitted signal and $\sigma_{w}^{2}=E\left[|w(k)|^{2}\right]$ denotes the energy of the AWGN.

Let the Log-Likelihood Function $\Lambda(\theta, \varepsilon)$ be defined as

$$
\begin{aligned}
\Lambda(\theta, \varepsilon) & \sum_{k=\theta}^{\theta+L-1}\left(\begin{array}{l}
\left|r(k) r^{*}(k+N)\right| \cos \left(2 \pi \varepsilon+\angle r(k) r^{*}(k+N)\right) \\
-\frac{\rho}{2}\left(|r(k)|^{2}+|r(k+N)|^{2}\right)
\end{array}\right)
\end{aligned}
$$

where $\rho=\frac{S N R}{S N R+1}$ is a constant related to the Signal-to-Noise Ratio (SNR) and $\angle$ denotes the phase. The ML method estimates the time delay and frequency offset as Equation(5) and Equation(6) respectively.

$$
\begin{aligned}
& \max _{(\theta, \varepsilon)} \Lambda(\theta, \varepsilon) \\
&= \max _{\theta} \Lambda\left(\theta, \hat{\varepsilon}_{\mathrm{ML}}(\theta)\right) \\
&=\left.\sum_{k=\theta}^{\theta+L-1}(\mid r(k) r(k+N))^{*} \mid-\frac{\rho}{2}\left(|r(k)|^{2}+|r(k+N)|^{2}\right)\right) \\
& \hat{\theta}_{\mathrm{ML}} \arg \max _{\theta} \sum_{k=\theta}^{\theta+L-1}\left(\left|r(k) r^{*}(k+N)\right|-\frac{\rho}{2}\left(|r(k)|^{2}+|r(k+N)|^{2}\right)\right) \\
& \hat{\varepsilon}_{\mathrm{ML}}=-\frac{1}{2 \pi} \angle \sum_{k=\hat{\theta}_{\mathrm{ML}}} r(k) r^{*}(k+N)
\end{aligned}
$$

In the ML merthod, the performance of the estimated time delay and frequency offset depend heavily on the length of the inserted CP. Table 1 lists the parameters for the ML method in [3] under different lengths of CP. Figures 2 and $\mathbf{3}$ are the corresponding time and frequency simulation results. According to the results, we can note that if we increase the length of the CP, the Mean Square Errors (MSE) for both the time and frequency offsets can be improved.

Based on above observation, if we use a preamble with two repetitions to conduct the synchronization, the ML method should perform well. A simple extension for the
ML method can be depicted in Figure 4.

Table 1. Simulation parameters for the ML method.

\begin{tabular}{lc}
\hline \multicolumn{1}{c}{ Modulation Type } & QPSK \\
\hline Number of Subcarriers , N & 256 \\
Cyclic Prefix Length , L & $4 、 6 、 8$ \\
Delay Time(sample) , & $16 、 32$ \\
Normalized Frequency Offset , & 128 \\
Channel Model & 0.4 \\
Monte Carlo & AWGN \\
\hline
\end{tabular}

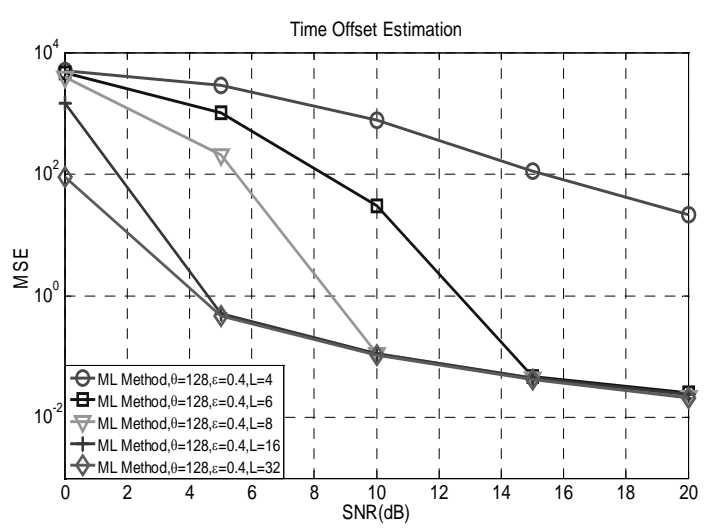

Figure 2. MSE of the time delay.

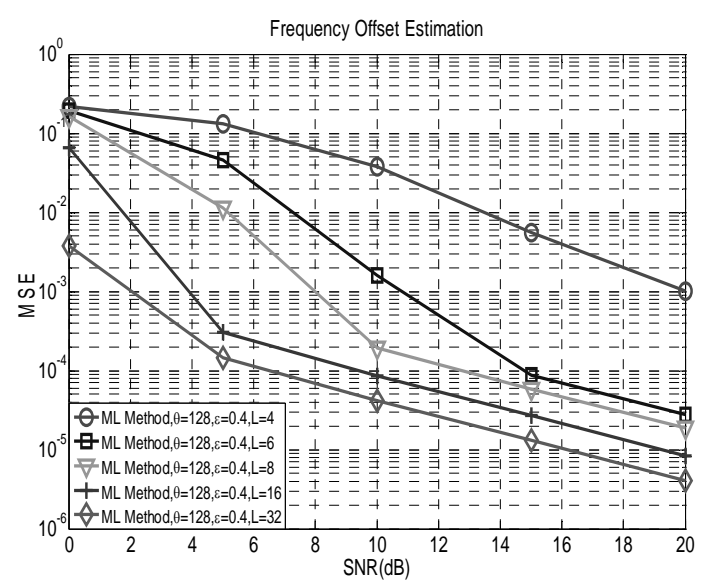

Figure 3. MSE of the frequency offset.

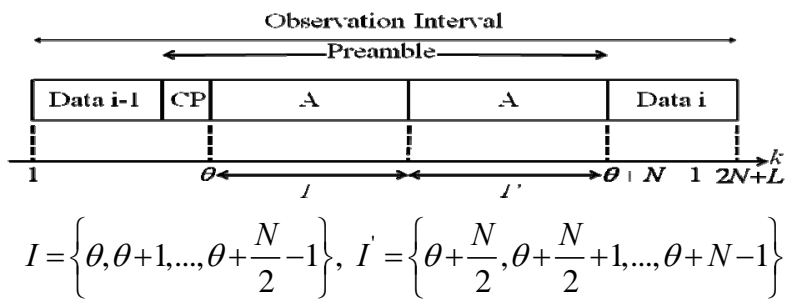

Figure 4. Preamble with two repetitions. 
In Figure 4, we construct a preamble with two repetitions to conduct the ML estimation. The ML method calculates the correlation between the defined intervals I and I'. However, a serious plateau problem will arise. This problem comes from the inherent correlation properties of the preamble with two or multiple repetitions. The plateau problem can be easily solved if we calculate the cross-correlation in an overlap manner. Figure $\mathbf{5}$ is an example that the intervals I and I' are overlapped. In this case, the length of the overlap region is exactly to $\mathrm{L}$. In the simulation part, we will demonstrate that with the help of the overlap region, the plateau problem can be efficiently reduced. The corresponding time delay and frequency offset estimations can be formulated as in Equation(7) and Equation(8).

$$
\begin{gathered}
\hat{\theta}_{\text {overap }} \\
\arg \max _{\theta_{2}} \sum_{k=\theta_{2}}^{\theta_{2}+\frac{N}{2}+L-1}\left(\left|r(k) r^{*}\left(k+\frac{N}{2}\right)\right|-\frac{\rho}{2}\left(|r(k)|^{2}+\left|r\left(k+\frac{N}{2}\right)\right|^{2}\right)\right) \\
\hat{\varepsilon}_{\text {overap }}=-\frac{1}{\pi} \angle \sum_{k=\hat{\theta}_{\text {overlap }}}^{\hat{\theta}_{\text {overlap }}+\frac{N}{2}+L-1} r(k) r^{*}\left(k+\frac{N}{2}\right)
\end{gathered}
$$

The main difference between the Equations 5-6 with Equations 7-8 is the range for the cross-correlation calculation. The overlap concept can ease the plateau problem of synchronization if a preamble contains multiple repetitions. Besides, the performance of the synchronization can be further improved if we adopt the iteration operation for the preamble. For example, if the Part-A of the preamble in Figure $\mathbf{5}$ can be further constructed with two repetitions as illustrated in Figure 6. The preamble wilt multiple repetitions can be easily generated if we adopt the Constant Pilot Padding Method (CPPM) [9] proposed previously. Thereafter, we can conduct the following iterative steps to increase the synchronization precision for both the time and frequency.

Step.1 Calculate the ML estimation for the intervals ( $I$ and $I^{\prime}$ ) with N/2 separation in Figrue 7. In this case, the intervals $I$ and $I^{\prime}$ contain the overlap region with length equals to $\mathrm{L}$.

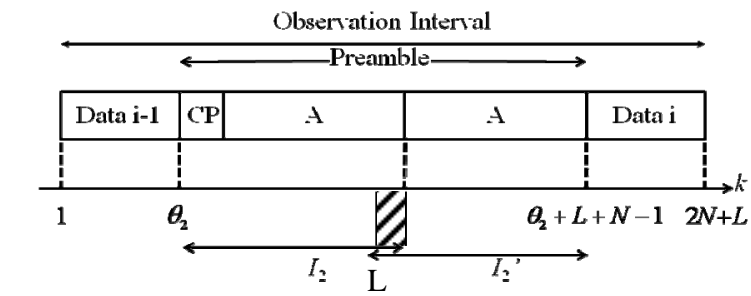

$I_{2}=\left\{\theta_{2}, \theta_{2}+1, \ldots, \theta_{2}+L+\frac{N}{2}-1\right\}, I_{2}^{\prime}=\left\{\theta_{2}+\frac{N}{2}, \theta_{2}+\frac{N}{2}+1, \ldots, \theta_{2}+L+N-1\right\}$

Figure 5. Overlap cross-correlation calculation.

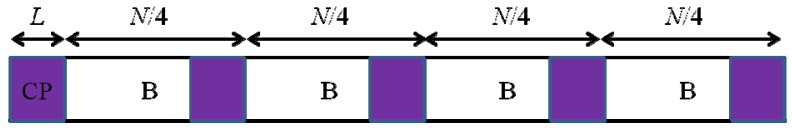

Figure 6. Preamble for iterative operations.

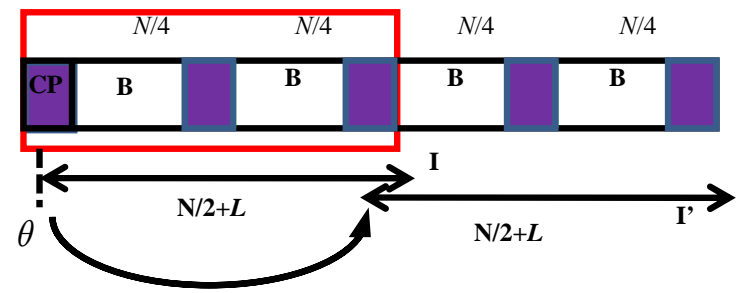

Figure 7. ML calculation for interval I and I'.

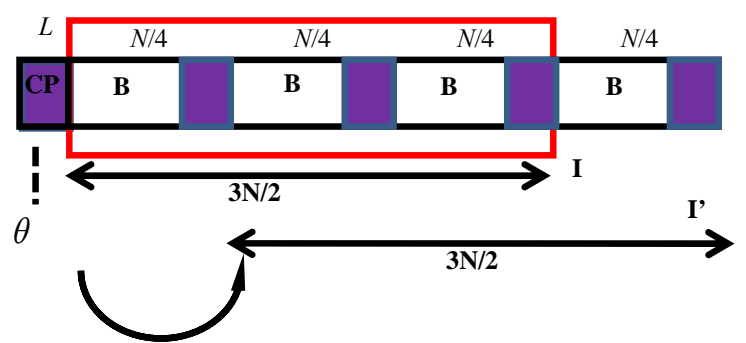

Figure 8. ML calculation for interval I and I'

Step 2 Calculate the ML estimation for the intervals ( $I$ and $I^{\prime}$ ) with $\mathrm{N} / 4$ separation in Figure 8. In this case, the intervals $I$ and $I^{\prime}$ contain the overlap region with length equals to $N / 2$.

Step 3 Calculate the estimated time and frequency offsets via the Equations 9-10. We can have more precise synchronization estimation which will be shown at the simulations in Section III.

$$
\begin{aligned}
\hat{\theta}_{\text {Iterative }}= & \arg \max _{\theta}\left\{\sum_{k=\theta}^{\theta+L+\frac{N}{2}-1}\left|\gamma_{1}(k)\right|\right\} \\
& +\left\{\sum_{k=\theta+L}^{\theta+L+\frac{3 N}{4}-1}\left|\gamma_{2}(k)\right|\right\} \\
& -\frac{\rho}{2}\left\{\left\{\sum_{k=\theta}^{\theta+L+\frac{N}{2}-1} \phi_{1}(k)\right\}+\left\{\sum_{k=\theta+L}^{\theta+L+\frac{3 N}{4}-1} \phi_{2}(k)\right\}\right\} \\
& \hat{\varepsilon}_{\text {Iterative }}=-\frac{1}{\pi} \angle \sum_{k=\hat{\theta}_{\text {Iterative }}}^{\hat{\theta}_{\text {Iterrative }}+L+\frac{N}{2}-1} \gamma_{1}(k)
\end{aligned}
$$

where

$$
\begin{aligned}
& \gamma_{1}(k)=r(k) r^{*}\left(k+\frac{N}{2}\right) \\
& \gamma_{2}(k)=r(k) r^{*}\left(k+\frac{N}{4}\right)
\end{aligned}
$$




$$
\begin{aligned}
& \phi_{1}(k)=|r(k)|^{2}+\left|r\left(k+\frac{N}{2}\right)\right|^{2} \\
& \phi_{2}(k)=|r(k)|^{2}+\left|r\left(k+\frac{N}{4}\right)\right|^{2}
\end{aligned}
$$

\section{Computer Simulations}

Table 2 lists the parameters to evaluate the proposed overlap concept. The delay time offset is 128 in this simulation. Figrue 9 is the Log-Likelihood function calculation if the two-repetition preamble conducts only non- overlapped cross correlation calculation, L/2-overlapped calculation and the L-overlapped cross correlation calculation respectively. Results show that if the length of overlap equals to or greater than the length of $\mathrm{CP}$, it can avoid the plateau problem efficiently. Figures $\mathbf{1 0}$ and $\mathbf{1 1}$ are the corresponding time and frequency offsets estimation for different SNR scenarios. With the help of the overlapped cross-correlation calculation, the proposed concept can predict the offsets correctly.

Table 3 lists the parameters to evaluate the proposed iterative method under the multipath fading channel. Path attenuations are according to the Vehicular-A [8] channel model. Figures 12 and $\mathbf{1 3}$ are the estimated time and frequency offsets. Results show with the proposed iterative method, the synchronization precision can be further improved for both the AWGN and multipath fading channels.

Table 2. Simulation parameters.

\begin{tabular}{lc}
\hline \multicolumn{1}{c}{ Modulation Type } & QPSK \\
\hline Number of Subcarriers & 256 \\
Cyclic Prefix Length & 32 \\
Delay Time(sample) & 128 \\
Normalized Frequency Offset & 0.4 \\
Channel Model & AWGN \\
Monte Carlo & 10000times \\
\hline
\end{tabular}

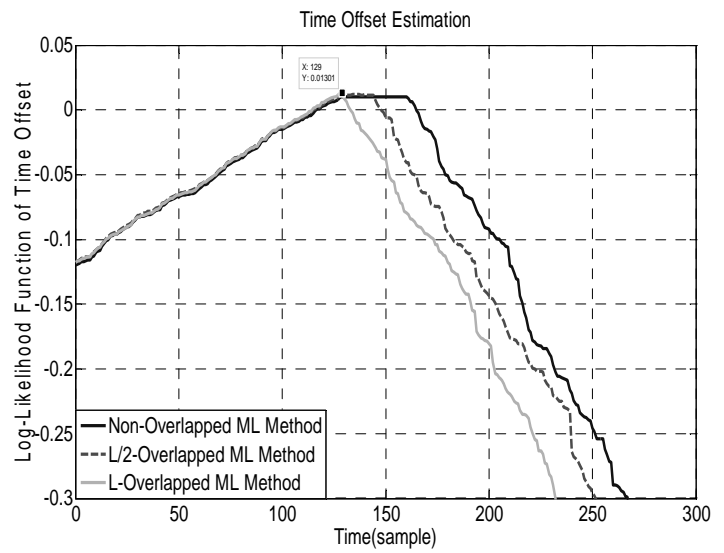

Figure 9. Log-Likelihood function calculation.

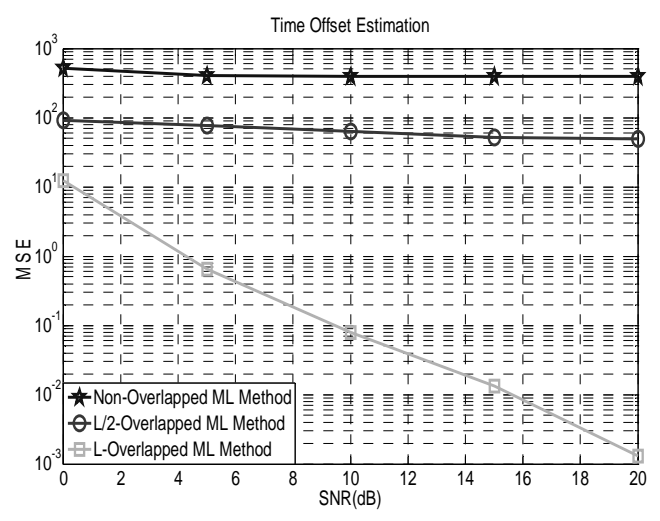

Figure 10. MSE of the timing offset estimation.

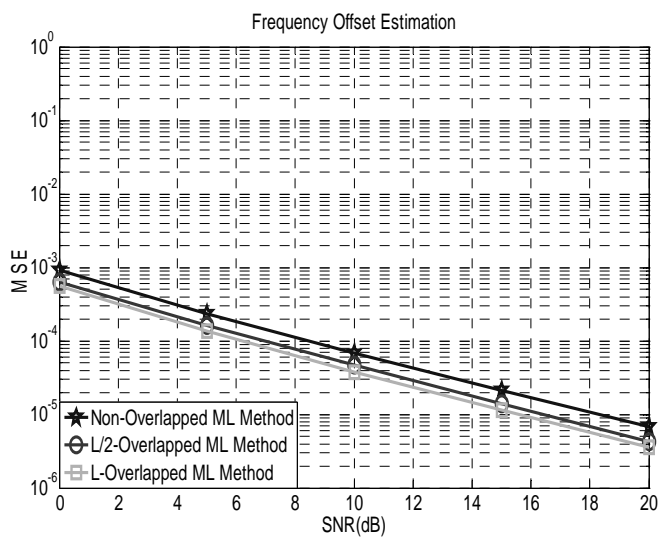

Figure 11. MSE of the frequency offset estimation.

Table 3. Simulation parameters.

\begin{tabular}{lccc}
\hline \multicolumn{1}{c}{ Modulation Type } & \multicolumn{3}{c}{ QPSK } \\
\cline { 2 - 4 } Number of Subcarriers & \multicolumn{3}{c}{256} \\
$\begin{array}{l}\text { Cyclic Prefix Length } \\
\text { Delay Time(sample) }\end{array}$ & 128 & 132 & 136 \\
Path Gain(dB) & 0 & -1 & -9 \\
Normalized Frequency Offset & \multicolumn{2}{c}{0.4} \\
Channel Model & AWGN 3Paths Rayleigh Fading Channel \\
Monte Carlo & \multicolumn{3}{c}{ 10000times } \\
\hline
\end{tabular}

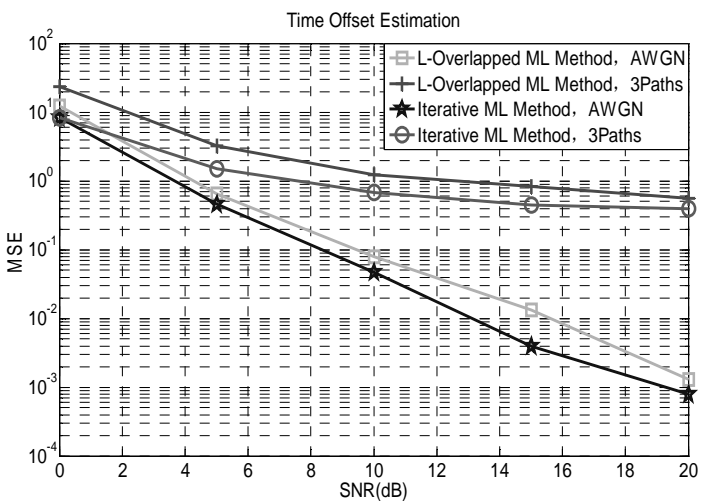

Figure 12. MSE of the time offset estimation. 


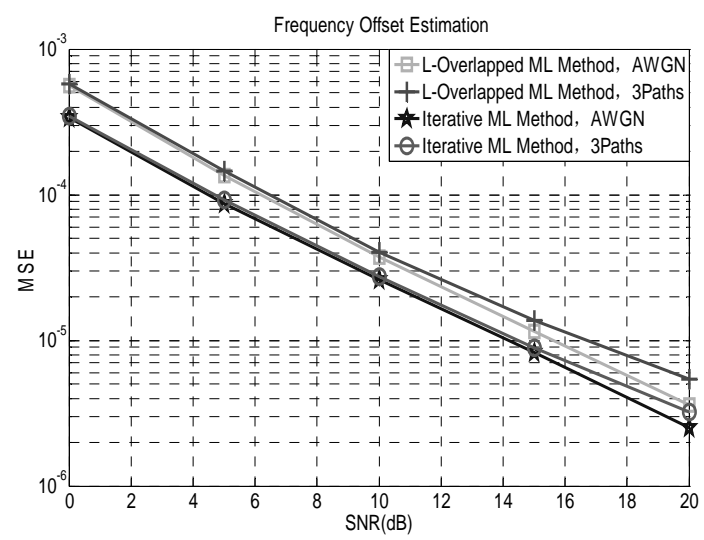

Figure 13. MSE of the frequency offset estimation.

\section{Conclusions}

In this paper, we propose an iterative ML method for synchronization. We adopt the overlap concept to reduce the plateau problem. Actually, any form of preamble contains a two-repetition or multiple-repetition structure can conduct the proposed iterative method easily. Simulation results show that the proposed method can predict the time delay and frequency offset correctly even under the multipath fading scenarios.

\section{REFERENCES}

[1] T. M. Schmidl and D. C. Cox, "Robust Frequency and Timing Synchronization for OFDM," IEEE Transactions on Communication, Vol. 45, No. 12, 1997, pp.1613-1621. doi:10.1109/26.650240
[2] Z. S. Pang and X. M. Li, “A Novel Synchronization Algorithm for OFDM System Based on Training Sequence Added Scramble Code," IEEE Communications Technology and Applications (ICCTA), 2009

[3] J. J. van de Beek, M. Sandell and P. O. Borjesson, "ML Estimation of Time and Frequency Offset in OFDM Systems,” IEEE Transactions on Signal Processing, Vol. 45, No.7, 1997, pp.1800-1805. doi;10.1109/78.599949

[4] H. Minn, M. Zeng and V. K. Bhargava, "On Timing Offset Estimation for OFDM Systems," IEEE Communications on Letters, Vol. 4, No. 7, 2000, pp. 242-244. doi;10.1109/4234.852929

[5] S. D. Ma, X. Y. Pan, G. H. Yang and T. S. Ng, "Blind Symbol Synchronization Based on Cyclic Prefix for OFDM Systems," IEEE Transactions on Vehicular Technology, Vol. 58, No. 4, 2009, pp. 1746-1751.

doi;10.1109/TVT.2008.2004031

[6] Z. Q. He, Y. Han and X. Fu, "Frequency Synchronization Algorithm Based on PN Sequence,"4th International Conference on Wireless Communications, Networking and Mobile Computing, 2008.

[7] H. Hwang and H. Park, "A Timing Synchronization Design In OFDM Systems,” IEEE International Workshop on Satellite and Space Communications, 2008, pp.290-293. doi;10.1109/IWSSC.2008.4656815

[8] 3GPP TS 25.101 V5.12.0, “Technical Specification Group Radio Access Network User Equipment (UE) Radio Transmission and Reception (FDD),” 2004.

[9] C. M. Li, H. C. Chen, P. J. Wang, J. C. Wu and I. T. Tang, "Timing Synchronization and Channel Estimation of a Constant Pilot Padding OFDM System," The 14th Asia-Pacific Conference on Communications (APCC2008) Tokyo, Japan. 